\title{
Genetically engineered recombinant adenovirus expressing interleukin-2 for hepatocellular carcinoma therapy
}

\author{
YUNPENG SUN, HUANHUAN WU, GANG CHEN, XIAMING HUANG, \\ YUNFENG SHAN, HONGQI SHI, QIYU ZHANG and YIHU ZHENG
}

\begin{abstract}
Department of Hepatobiliary Surgery, The First Affiliated Hospital of Wenzhou Medical University, Wenzhou, Zhejiang 325015, P.R. China
\end{abstract}

Received November 24, 2015; Accepted November 17, 2016

DOI: $10.3892 / \mathrm{mmr} .2017 .7922$

\begin{abstract}
Regulatory and effector $\mathrm{T}$ cells possess immunological cytotoxicity for tumor cells in the tumor microenvironment during tumor progression and are the primary suppressors inhuman cancer therapy. Interleukin-2 (IL-2) is an anticancer cytokine, which triggers human innate and adaptive immunity by stimulating $\mathrm{T}$ cell propagation and lymphocyte infiltration into tumor sites. IL-2 has been used successfully for cancer therapy. Recombinant adenovirus expressing IL-2 (rAd-IL-2) injection is a gene therapy agent that may improve prognosis of hepatocellular carcinoma (HCC) patients. In the present study, the ability of IL-2 to stimulate an immune response and the ability of recombinant adenovirus to inhibit tumor cell growth in HCC was investigated in a $\mathrm{HCC}$ tumor model. It was demonstrated that the regulatory and effector cell-mediated tumor suppression by antitumor cluster of differentiation (CD) $4^{+}$and $\mathrm{CD}^{+}$ T cells stimulated by rAd-IL-2 is tumor-specific. Furthermore, rAd-IL-2 significantly stimulated tumor-specific cytotoxic $\mathrm{T}$ lymphocyte responses, increased interferon- $\gamma$ release and enhanced antitumor immunity by inducing $\mathrm{CD}^{+}$and $\mathrm{CD}^{+}$ $\mathrm{T}$ cell recruitment into the tumor, and additionally induced memory to protect tumor-bearing mice against tumor challenge. Treatment with rAd-IL-2 led to tumor regression and long-term survival of mice in the 120-day treatment period. Tumor challenge experiments demonstrated that rAd-IL-2 induced memory, protecting against reinfection. In conclusion, rAd-IL-2 may promote tumor-associated effector and regulatory $\mathrm{T}$ cell expansion and may be a potential therapeutic agent for clinical immunotherapy application in the treatment of cancer.
\end{abstract}

Correspondence to: Dr Yihu Zheng, Department of Hepatobiliary Surgery, The First Affiliated Hospital of Wenzhou Medical University, 278 Ouhai Road, Wenzhou, Zhejiang 325015, P.R. China E-mail: tianhuismile@163.com

Key words: interleukin-2, recombinant adenovirus expressing interleukin-2, hepatocellular carcinoma, immunoregulation, immunotherapy, cluster of differentiation 4 , cluster of differentiation 8 , cytotoxic $\mathrm{T}$ lymphocytes

\section{Introduction}

Hepatocellular carcinoma (HCC) is the predominant form of primary liver cancer, which has a high recurrence, leading to high mortality rates following radiotherapy, chemotherapy and surgery (1). HCC has the greatest incidence among human cancers; however, therapeutic strategies remain limited, particularly for patients with advanced late-stage HCC (2). Currently, the common clinical therapies of surgery, chemotherapy and radiotherapy result in only a modest efficacy, and are associated with severe side-effects during and post-treatment. Therefore, novel clinical therapies with enhanced efficacy and reduced adverse effects are urgently required to prolong the survival of patients with HCC $(3,4)$. Tumor immunotherapy is a novel anticancer therapy strategy, which combines potential efficacy with no or few treatment-associated adverse events; this strategy may lead to improved treatment for HCC patients $(5,6)$. Previous studies on tumor immunotherapy have identified numerous candidates through target antigen presenting molecules and bioinformatics, which have revealed numerous methods by which the immune response may target tumor cells $(7,8)$. The present study demonstrated that mice with HCC benefited from immunotherapy with recombinant adenovirus expressing interleukin-2 (rAd-IL-2), which suggested that rAd-IL-2 immunotherapy may provide therapeutic advantages and improve the quality of life for patients with HCC.

Interleukin-2 (IL-2) is a pleiotropic and multifunctional cytokine, which is important for adaptive immune systems (9). IL-2 treatment was the first immunotherapy approved by the US Food and Drug Administration for use in melanoma (10). IL-2 serves a pivotal role in T-cell activation and effector function, including proliferation and cytotoxicity (11). IL-2 may stimulate the propagation of lymphocytes and induce cytotoxic T lymphocyte (CTL) and lymphokine-activated killer (LAK) cell responses against numerous tumor cells (12). Furthermore, IL-2 influences memory T cell homeostasis via regulation of memory $\mathrm{T}$ cell numbers, driving the generation of antigen-specific $\mathrm{T}$ cells and promoting the survival of memory cluster of differentiation (CD) $8^{+} \mathrm{T}$ cells (13). Therefore, it has been used for cancer immunotherapy. In addition, previous studies have reported that IL-2 gene therapy enhances the 
efficacy of other anticancer agents by stimulating the immune system to produce tumor-specific immune cells (14).

Previous studies have indicated that gene therapy is a potential clinical strategy with improved efficacy for HCC, and may be a potential adjuvant for other cancer therapies $(15,16)$. The adenovirus vector is the most widely used and adenovirus-mediated delivery of functional genes or polypeptides into tumor cells is well understood (17). These results have led clinicians to use immunotherapy, including inhibition of oncogenes and restoration of tumor-suppressor genes, anti-angiogenesis, and virotherapy to treat patients with HCC (18). Therefore, the present study may increase the efficacy of immunotherapy for HCC by delivering the IL-2 gene using a recombinant adenovirus vector, which may be combined with alternative therapeutic options as an adjunct therapy.

In the present study, a mouse model of HCC was used to study the therapeutic effects of rAd-IL-2. The effects of IL-2 immunotherapy were consistent with the majority of studies that revealed therapeutic effects, and were additionally demonstrated in HCC mice treated with rAd-IL-2. rAd-IL-2 gene therapy has reached preclinical development in the treatment of HCC. The present study aimed to investigate the impact of rAd-IL-2 on HCC apoptosis, prognosis and treatment, and its effect on lymphocyte infiltration, toxicity and memory. The results of the present study may provide insight into the pathophysiology of HCC, and may suggest a potential therapeutic strategy for HCC.

\section{Materials and methods}

Ethics statement. The present study was carried out in strict accordance with the recommendations in the Guide for the Care and Use of Laboratory Animals, produced by the National Institutes of Health (Bethesda, MD, USA), and was approved by the Committee on the Ethics of Animal Experiments Defense Research of The First Affiliated Hospital of Wenzhou Medical University (Wenzhou, Zhejiang, China). All surgery and euthanasia (performed following 120 days due to tumor progression) were designed to minimize suffering.

Cell culture. HepG2 human liver carcinoma cells, H22 mouse hepatoma cells and HEK293 human embryonic kidney cells were purchased from the American Type Culture Collection (Manassas, VA, USA) and cultured in Dulbecco's modified Eagle's medium (Gibco; Thermo Fisher Scientific, Inc., Waltham, MA, USA) containing $10 \%$ fetal bovine serum (Gibco; Thermo Fisher Scientific, Inc.).

Construction of recombinant adenovirus. The expression system of Adeno-X (Shanghai Rebiosci Biotechnology Co., Ltd., Shanghai, China) was used to construct the recombinant adenovirus virion, according to the manufacturer's protocol. The DNA sequence encoding 402 basic group of human IL-2, including the cell-penetrating peptide, was amplified by polymerase chain reaction (PCR) from pMD-18-IL-2 and subcloned into the pAd-X plasmid to produce rAd-IL-2. The following primers were used for PCR: IL-2 forward, 5'-CAA AGGTGGATCAGATTCAAG-3' and reverse, 5'-GGTGAG CATTATCACCCAGAA-3'; $\beta$-actin forward, 5'-GAGGGC
AGAATCATCACGAAGT-3' and reverse, 5'-TGAGAGATC TGGTTCCCGAAAC-3'. The thermocycling conditions were as follows: $95^{\circ} \mathrm{C}$ for $30 \mathrm{sec}$, followed by 45 cycles of $95^{\circ} \mathrm{C}$ for $5 \mathrm{sec}$ and $60^{\circ} \mathrm{C}$ for $40 \mathrm{sec}$. PCR and gene sequencing were used to select the correct clone, which was translated into $E$. coli. The recombinant adenoviruses were generated by transfecting HEK 293 cells, which were then cultured. Recombinant viruses were purified as previously described (19). Adenovirus titers were determined by $50 \%$ cell culture infectious dose, as plaque-forming units $(\mathrm{pfu}) / \mathrm{ml}$.

Analysis of rAd-enhanced green fluorescent protein (EGFP) expression in the constructed adenovirus using rAd-IL2 miRNA-short hairpin-RNA (miRsh). H22 cells $\left(1 \times 10^{5}\right)$ and HepG2 cells $\left(1 \times 10^{5}\right)$ were infected with $\mathrm{rAd}-\mathrm{IL}-2 \mathrm{miR}$ sh with a multiplicity of infection (MOI) of $10 \mathrm{pfu} / \mathrm{cell}$. The cells were then incubated with anti-EGFP (1:1,000; cat. no. VPY0002; ChangshaYingrun Biotechnology Co., Ltd., Hunan, China) at $37^{\circ} \mathrm{C}$ for $24 \mathrm{~h}$, and EGFP expression was examined at $48 \mathrm{~h}$ following infection using an Olympus fluorescence imaging system (BX63; Olympus Corporation, Tokyo, Japan; fluorescent filter set at an excitation of 450-490 nm).

Animal experiments. Specific pathogen-free female BALB/c nude mice $(n=120$; age, 6 weeks) were purchased from Harbin Veterinary Research Institute (Harbin, China). All animals were maintained under pathogen-free conditions at a constant temperature and humidity $\left(22 \pm 1^{\circ} \mathrm{C} ; 50 \%\right)$, and a 12-h light/dark cycle, with $<5$ per cage and ad libitum access to food and water. $\mathrm{H} 22$ cells $\left(1 \times 10^{6}\right)$ were injected into the right flank of female BALB/c nude mice in a total volume of $200 \mu$ l. rAd-IL-2 therapy was initiated when tumor diameters reached 6-8 $\mathrm{mm}$ on day 7 after tumor inoculation. Mice with HCC were randomly divided into three groups $(n=15$ in each group) and injected intratumorally with $2 \times 10^{7}$ pfu rAd-IL-2, EGFP (Shanghai Rebiosci Biotechnology Co., Ltd.) or PBS. The treatment was repeated five times at intervals of two days. Tumor diameters were recorded once every two days and tumor volume was calculated using the following formula: $0.52 \mathrm{x}$ smallest diameter ${ }^{2} \mathrm{x}$ largest diameter.

Splenocyte collection and CTL responses. Splenocytes were obtained from the spleens of euthanized animals. A total of 15BALB/c mice treated with rAd-IL-2, as aforementioned, were sacrificed by a subcutaneous injection of $200 \mathrm{mg} / \mathrm{kg}$ of pentobarbital sodium. Red blood cells were lysed in radioimmunoprecipitation buffer (Shanghai Rebiosci Biotechnology Co., Ltd.). The cell suspensions were washed three times with PBS and incubated with $\mathrm{H} 22$ cells inactivated by $30 \mu \mathrm{g} / \mathrm{ml}$ mitomycin (Sbjbio, Nanjing, China). The secretion of interferon- $\gamma($ IFN- $\gamma)$ was evaluated by ELISA in the supernatants after culture for $72 \mathrm{~h}$. ELISA was performed using a sandwich ELISA kit to determine the IFN- $\gamma$ (cat. no. ab193969; Abcam, Cambridge, UK) and IL-2 (cat. no. ab208207; Abcam) expression levels in the supernatants according to the manufacturer's instructions. In addition, all T cells $\left(1 \times 10^{6}\right)$ were purified from splenocytes as previously described (20) and co-cultured with fresh $\mathrm{H} 22$ cells for $4 \mathrm{~h}$ at effector:target ratios of 10:1, 20:1 and 40:1. Specific CTL activity against the target cells was determined by MTT cytotoxicity assays 


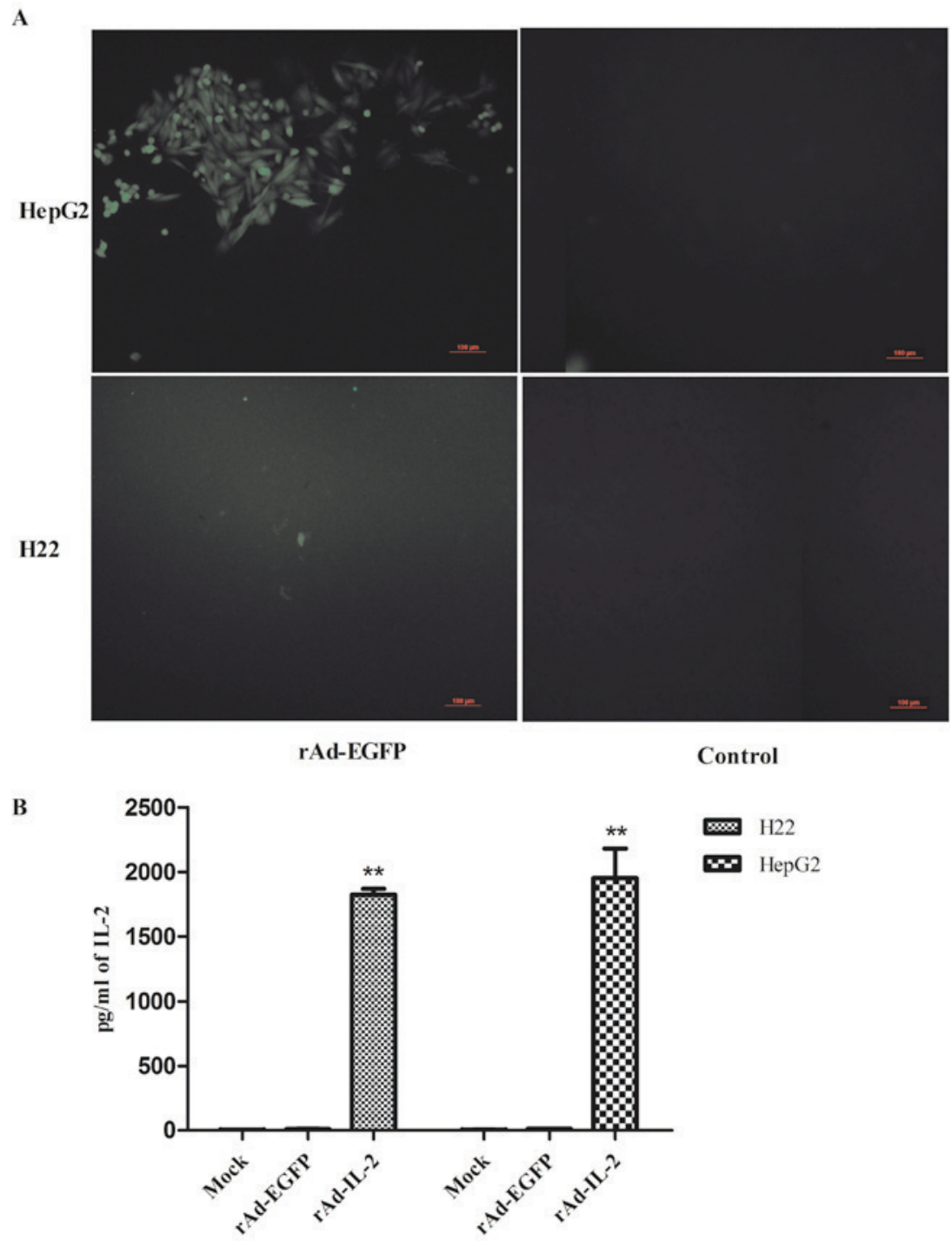

Figure 1. EGFP Expression in rAd-EGFP-infected $\mathrm{HepG} 2$ and $\mathrm{H} 22$ cells. The HepG2 and $\mathrm{H} 22$ cells were infected with rAd-EGFP at a multiplicity of infection of 40. (A) EGFP expression in rAd-EGFP-infected HepG2 and H22 cells was observed under a fluorescence microscope (scale bar, $100 \mu \mathrm{m}$ ). (B) IL-2 expression in HepG2 and $\mathrm{H} 22$ cells supernatants was detected using ELISA. ${ }^{* *} \mathrm{P}<0.01$ vs. Mock (untreated control cells). EGFP, enhanced green fluorescent protein; rAd, recombinant adenovirus; IL-2, interleukin-2.

as previously described (21). For the control group, T cells $\left(1 \times 10^{6}\right)$ were cultured using commercial IL-2 $(100 \mu \mathrm{g} / \mathrm{ml}$; cat. no. AZ-013, Shanghai Jianglai Bioscience, Co., Ltd., Shanghai, China) for $4 \mathrm{~h}$ at $37^{\circ} \mathrm{C}$. The negative control group was cultured in culture medium only without any treatments.

Flow cytometric analysis. Cell suspensions from tumors of treated mice were prepared for flow cytometric analysis. Cells were carefully suspended in the residual volume of staining buffer and then $200 \mu \mathrm{l}$ of freshly prepared cold fixation buffer (Shanghai Haoran Biological Technology Co., Ltd., Shanghai, China) was added. Cells were fixed with $70 \%$ ethyl alcohol (Shanghai Haoran Biological Technology Co., Ltd.) for $30 \mathrm{~min}$ at $4^{\circ} \mathrm{C}$ in the dark. Then, the sample was centrifuged at $1,000 \mathrm{xg}$ for $5 \mathrm{~min}$ at room temperature and the fixative was removed. To permeabilize the cells, the cell pellet was suspended in $200 \mu \mathrm{l}$ of freshly prepared, pre-warmed $\left(37^{\circ} \mathrm{C}\right)$ permeabilization buffer (Shanghai Haoran Biological Technology Co., Ltd.) and the cells were then incubated for $30 \mathrm{~min}$ at $37^{\circ} \mathrm{C}$ in the dark. The cells were subsequently centrifuged at $1,000 \mathrm{x}$ for $5 \mathrm{~min}$ at room temperature and the buffer was removed. To wash the cells, $200 \mu 1$ of PBS was added to each tube $\left(1 \times 10^{5}\right)$, centrifuged at $1,000 \mathrm{x}$ g for $5 \mathrm{~min}$ at room temperature and the buffer was removed. Then, the tumor cells were labeled with FITC-conjugated CD3 (cat. no. ab16669; Abcam) and phycoerythrin-conjugated CD45 (cat. no. ab10550; Abcam) plus peridinium chlorophyll protein-conjugated CD4 (cat. no. ab19514; Abcam) and CD8 (cat. no. ab25477; Abcam) antibodies to assess the frequency of CD4 and CD8 cell subsets in total infiltrated immune cells. The stained cells were analyzed using a Becton Dickinson FACScan flow cytometer (BD Biosciences, Franklin Lakes, NJ, USA) and WinMD I software (version 2.9; BD Biosciences).

Statistical analysis. All data are expressed as the mean \pm standard deviation. Analysis was performed using SPSS software version 20.0 (IBM Corp., Armonk, NY, USA). Unpaired data was analyzed using a Student's t-test. Multiple 
A

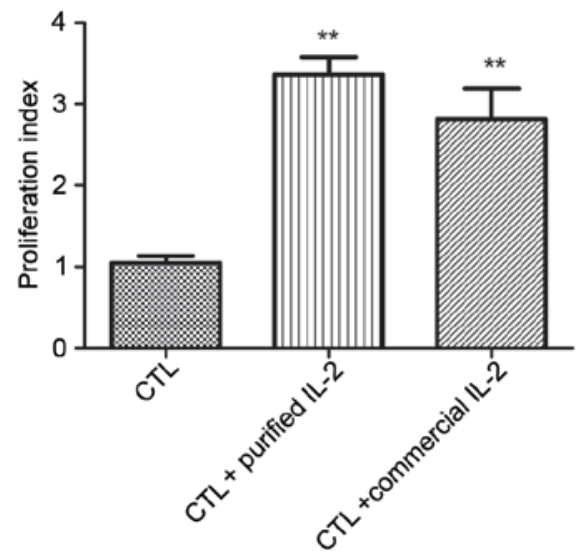

B

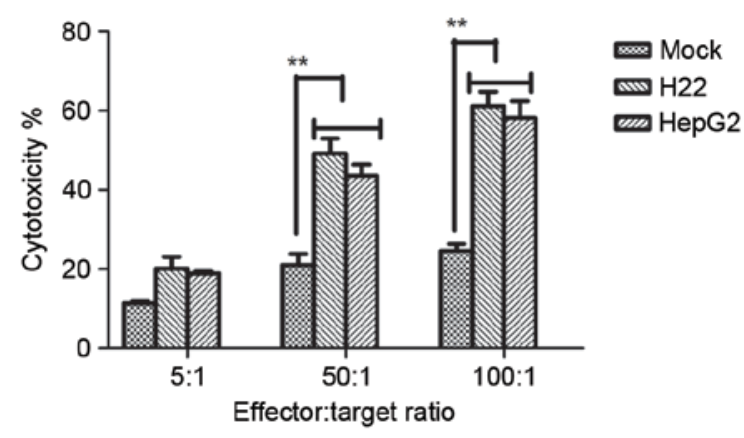

Figure 2. Characterization and biological activities of IL-2expressed by rAd-IL-2-infected tumor cells. (A) CTL proliferation was stimulated by IL-2expressed by rAd-IL-2-infected HepG2 cells. ${ }^{* *} \mathrm{P}<0.01$ vs. CTL. (B) The purified IL-2 enhanced the cytotoxicity of immune cells against $\mathrm{H} 22$ and HepG2 cells. ${ }^{* *} \mathrm{P}<0.01$. Data are expressed as the mean \pm standard deviation. IL-2, interleukin-2; rAd, recombinant adenovirus; CTL, cytotoxic T lymphocyte; Mock, untreated controls cultured in culture medium only.
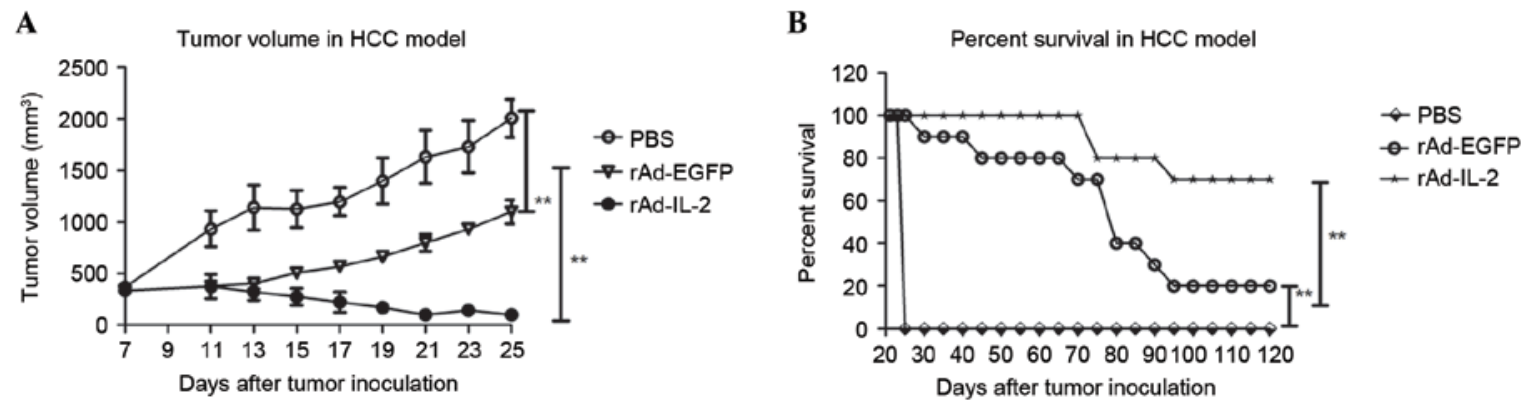

Figure 3. Tumor growth inhibition and long-term survival of H22-bearing BALB/c nude mice. Mice with HCC were injected intratumorally with rAd-IL-2, rAd-EGFP or PBS. (A) The tumor volumes of each experimental mouse were recorded every two days. (B) Survival of mice was assessed up to 120 days. Data are expressed as the mean \pm standard deviation. ${ }^{* *} \mathrm{P}<0.01$. rAd, recombinant adenovirus; IL-2, interleukin-2; EGFP, enhanced green fluorescent protein; HCC, hepatocellular carcinoma.

groups were compared using one-way analysis of variance and a Tukey honest significant difference post hoc test. $\mathrm{P}<0.05$ was considered to indicate a statistically significant difference.

\section{Results}

Detection of recombinant IL-2 in rAd-IL-2-infected cells in vitro. To detect the expression of foreign genes, the expression of EGFP in $\mathrm{H} 22$ and HepG2 cells infected with rAd-EGFP were observed under a fluorescence microscope. Fig. 1A demonstrates that EGFP was observed in $\mathrm{H} 22$ and HepG2 cells, suggesting that foreign genes were efficiently expressed by tumor cells. To confirm the expression efficiency of IL-2 in rAd-IL-2-infected tumor cells, the expression of IL-2 in H22 and HepG2 cells infected with rAd-IL-2 was detected by ELISA using an anti-IL-2 antibody. The results presented in Fig. 1B revealed that IL-2 protein was expressed and secreted by tumor cells infected with rAd-IL-2.

Characterization of the IL-2 protein. To investigate the biological activity of IL-2 in rAd-IL-2-infected tumor cells, the proliferation of $\mathrm{T}$ cells was examined following treatment with IL-2 from rAd-IL-2-infected HepG2 cells. As presented in Fig. 2A, purified IL-2 from rAd-IL-2-infected tumor cells stimulated the proliferation of CTL cells compared with untreated cells. This indicated thattheIL-2 protein expressed by rAd-IL-2-infected tumor cells was biologically active. In addition, the cytotoxicity of splenocytes stimulated with IL-2 from rAd-IL-2-infected cells was assessed. As presented in Fig. 2B, IL-2 from HepG2 cells infected with rAd-IL-2 stimulated $\mathrm{T}$ cells to differentiate into LAK cells, resulting in increased cell death of HepG2 and $\mathrm{H} 22$ cells compared with the control group.

In vivo antitumor efficacy of rAd-IL-2. Subsequently, the antitumor effect of treatment with rAd-IL-2 was investigated in an HCC mouse model, using mice treated with PBS and rAd-EGFP as controls. Tumors in the PBS-treated group reached a volume of $2007.43 \pm 123.42 \mathrm{~mm}^{3}$ by day 25 after tumor incubation (Fig. 3A). Tumor growth increased in the rAd-EGFP-treated group, although the rate of growth was significantly reduced compared with the PBS-treated group $(\mathrm{P}<0.01)$. By contrast, the growth of tumors treated with rAd-IL-2 was significantly inhibited compared with the PBSand rAd-EGFP-treated groups $(\mathrm{P}<0.01)$. During the treatment period, no side-effects were observed except swelling at the injection site. Furthermore, the survival of mice was assessed long-term for 120 days. As presented in Fig. 3B, rAd-IL-2 
A

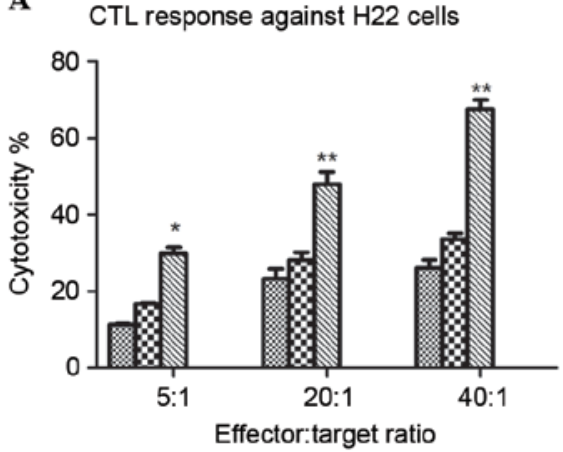

B Tumor-specific splenocyte IFN- $\gamma$ release
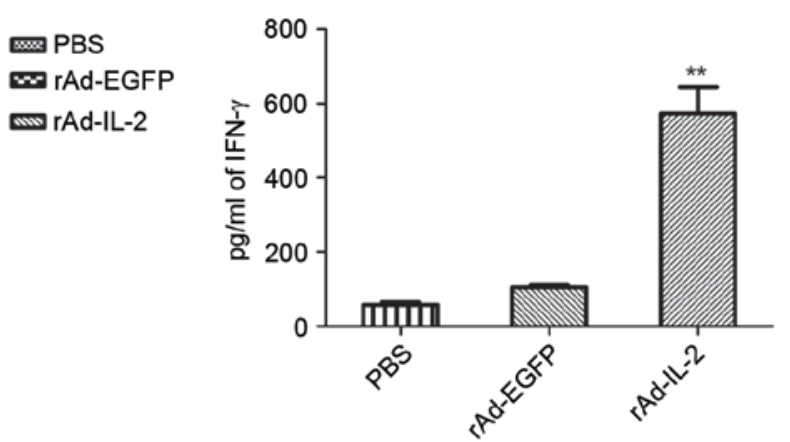

Figure 4. rAd-IL-2 virus enhanced immune responses against tumor cells ex vivo. (A) CTL responses against HCC cells. (B) Tumor-specific IFN- $\gamma$ secreted from stimulated splenocytes in the HCC model was detected by ELISA. Data are expressed as the mean \pm standard deviation. ${ }^{*}<<0.05$ and ${ }^{* *} \mathrm{P}<0.01$ vs. PBS rAd, recombinant adenovirus; IL-2, interleukin-2; EGFP, enhanced green fluorescent protein; CTL, cytotoxic T lymphocytes; HCC, hepatocellular carcinoma; IFN- $\gamma$, interferon- $\gamma$.
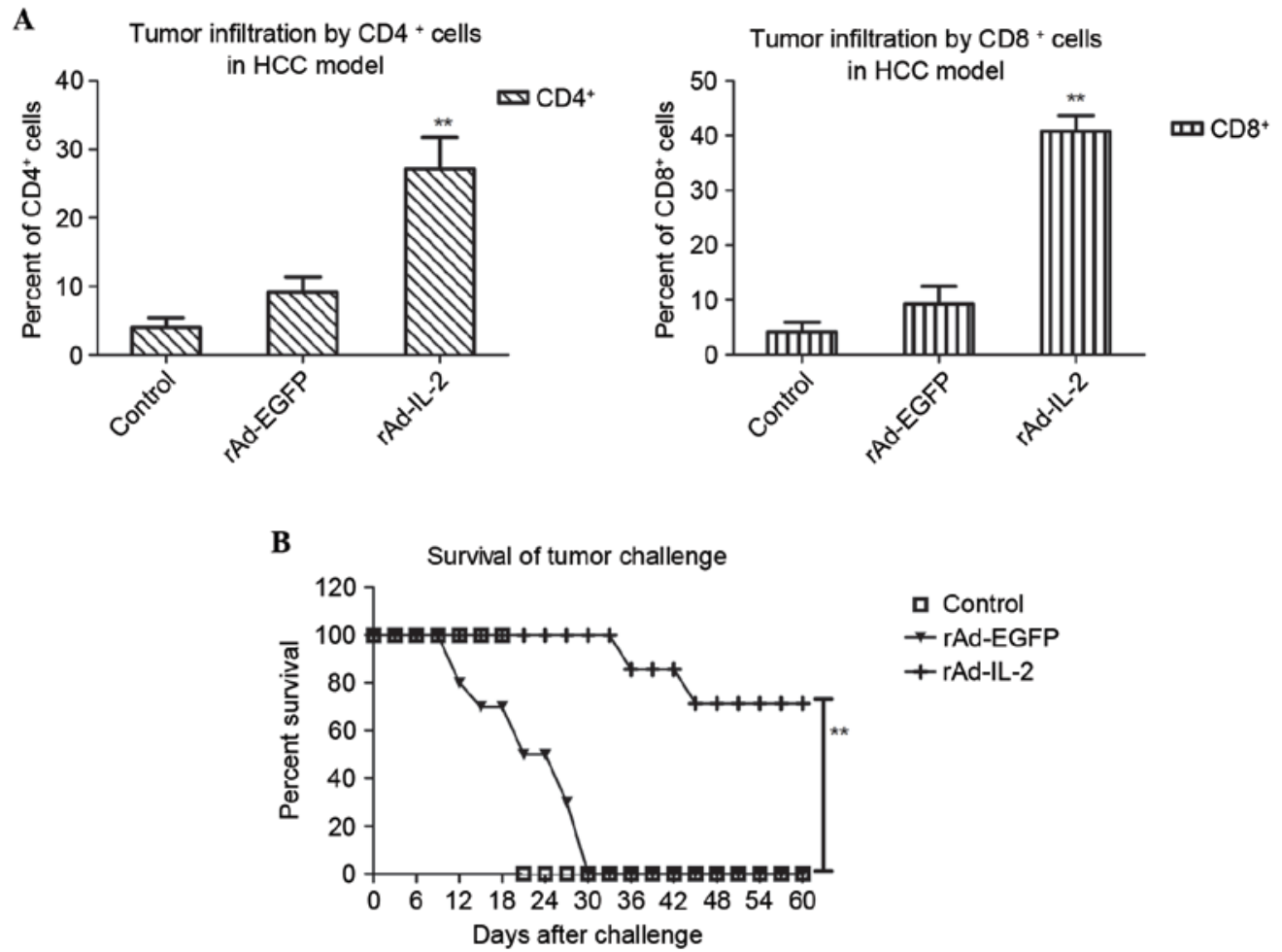

Figure 5. IL-2 expressed by rAd-IL-2 protected mice against tumor challenge in vivo. (A) The percentage of $\mathrm{CD}^{+}{ }^{+}$and $\mathrm{CD}^{+}$cells in tumors from $\mathrm{HCC}$ mice was analyzed by flow cytometry. (B) Tumor challenge with $\mathrm{H} 22$ cells was performed at 120 days. Data are expressed as the mean \pm standard deviation. ${ }^{* *} \mathrm{P}<0.01$ vs. control. IL-2, interleukin-2; rAd, recombinant adenovirus; CD, cluster of differentiation; HCC, hepatocellular carcinoma; EGFP, enhanced green fluorescent protein.

prolonged the survival of mice compared with control groups ( $n=15$ total mice; $n=10$ survived). These results indicate that the therapeutic agent rAd-IL-2 is effective in HCC tumor-bearing animals to eliminate the tumor cells, contributing to long-term and tumor-free or tumor-bearing survival.

rAd-IL-2 effectively induced toxicity. Adaptive immune responses are important for human cancer therapy. Therefore, the formation of adaptive immune responses against $\mathrm{H} 22$ cells was assessed in tumor-bearing mice treated with rAd-IL-2. As presented in Fig. 4A, mice treated with rAd-IL-2 developed a strong CTL response against $\mathrm{H} 22$ cells compared with the rAd-EGFP and PBS groups. The secretion of IFN- $\gamma$ by rAd-IL-2-treated mouse splenocytes was additionally examined. As presented in Fig. 4B, H22-bearing mice treated with rAd-IL-2 demonstrated greater IFN- $\gamma$ secretion compared with rAd-EGFP- and PBS-treated groups. These effects of rAd-IL-2 may contribute to the long-term survival of HCC mice.

Mice inoculated with rAd-IL-2 develop a memory response to H22 cells. Cancer recurrence is a common phenomenon in cancer therapy. To confirm whether HCC mice develop a memory response that protects mice against challenge with the same tumor cells, CD4 and CD8 expression and tumor 
challenge experiments were conducted after a 120-day survival period. As presented in Fig. 5A, tumors from rAd-IL-2-treatedmice contained a greater percentage of $\mathrm{CD}^{+}$ and $\mathrm{CD}^{+} \mathrm{T}$ cells compared with PBS- and rAd-EGFP treated mice, suggesting a greater immune response in these mice. Furthermore, tumor challenge with $1 \times 10^{5} \mathrm{H} 22$ cells injected on day 120 was performed to assess the memory response. As presented in Fig. 5B, mice treated with rAd-IL-2 demonstrated a memory response to $\mathrm{H} 22$ tumor cells, mediating prolonged survival of mice with HCC. Although H22-bearing mice treated with rAd-EGFP demonstrated slight immune memory against homologous tumor cells compared to the PBS-treated group, all mice in this group died by day 30 as a result of tumor recurrence. These results suggested that rAd-IL-2-treated mice developed immune responses and immune memory against tumor cells, which contributed to the long-term survival of mice and their partial protection from tumor recurrence.

\section{Discussion}

Previous studies have demonstrated that recombinant adenovirus constructed using the Adeno-X expression system is a suitable gene therapy vehicle in human cancers $(19,22)$. The adenovirus vector is one of the most widely used vectors in gene therapy applications for the treatment of diverse human diseases including cancer (23). Ye et al (24) reported that adenovirus-mediated gene therapy demonstrated beneficial preclinical outcomes by inducing apoptosis of tumor cells and inhibiting tumor angiogenesis, which led to $\mathrm{HCC}$ regression in a $\mathrm{HCC}$ tumor model. $\mathrm{Hu}$ et al (25) revealed that adenovirus-mediated expression of melanoma differentiation-associated-7 efficiently inhibited HCC growth. In addition, He et al (26) investigated the inhibition of HCC growth by adenovirus-mediated expression of human telomerase reverse transcriptase $\mathrm{COOH}-27$ terminal polypeptide in mice. However, there are few reports regarding immunotherapy using delivered recombinant adenovirus.

Immunotherapy in combination with other therapeutic agents has demonstrated clear benefits in animal models of different human cancers (27-29). Treatments with antineoplastic agents and immunotherapy have been demonstrated to be effective therapies for tumor cells, targeting specific recognition molecules (30-33). Currently, numerous immunotherapy agents against cancer are being assessed in preclinical studies. The immunotherapy agents, in which an antibody or interleukin is inserted into a gene expression vector, have revealed positive outcomes in patients with advanced tumors in clinical trials (34). The establishment of an effective gene delivery system is crucial for gene therapy. The present study combined an oncolytic adenovirus-mediated gene therapy system with IL-2 immunotherapy to treat HCC-bearing mice.

The antitumor activity of IL-2 has been demonstrated in human cancer therapy $(35,36)$. The application of IL-2 may improve the microenvironment of tumors or stimulate the immune system of mice. IL-2 may induce tumor cell eradication and prevent tumor recurrence by stimulating tumor-specific immune cells and improving the immunogenicity of tumor cells to enhance cytotoxic lymphocyte infiltration (37). The present study supports this hypothesis. It has additionally been reported that IL-2 is important for the expansion of immune memory T cells (38). The results of the present study indicated that memory $\mathrm{T}$ cells significantly contribute to long-term survival and tumor challenge through IL-2-mediated effects on the immune response. Furthermore, $\mathrm{CD}^{+} \mathrm{T}$ cells are an essential part of the adaptive immune system against intracellular pathogens and cancer, and IL-2 is an important molecule for $\mathrm{CD}^{+} \mathrm{T}$ cell responses. The results of the present study indicated that IL-2 protected long-term surviving mice by offering protection to certain animals.

The present study revealed that the CTL responses were significantly enhanced inrAd-IL-2-treated mice compared with rAd-EGFP- and PBS-treated controls. The enhanced cytotoxic sensitivity of tumor cells may have contributed to the long-term survival of mice. The expression of IL-2 by rAd-IL-2-infected tumor cells resulted in greater percentages of $\mathrm{CD}^{+}$and $\mathrm{CD}^{+}$cells in tumors from mice. Thus, promoting apoptosis via cytokine induction of killer cancer cells may be crucial in cancer treatment. Previous evidence has revealed that IL-2 expressed by Newcastle disease virus is an efficient anti-cancer agent (39). Although HCC cells are insensitive to apoptosis induced by the CTL response (39), the results of the present study revealed an improved effect when IL-2 was expressed by adenovirus. The expression system was tested using EGFP reporter assays. The results demonstrated that EGFP was detected in late onset infection and MOI cell lines infected with rAd-IL-2. Notably, the volume of tumors was significantly inhibited in the rAd-IL-2-treated mice compared with PBS- and rAd-EGFP-treated controls, suggesting that a greater effect was induced by IL-2 expressed by rAd-IL-2 in vivo. Therefore, the therapeutic effects against $\mathrm{HCC}$ cells in mice were achieved through treatment with rAd-IL-2.

The present study revealed that $\mathrm{AAd}-\mathrm{IL}-2$ treatment of mice with HCC stimulated T cell proliferation and the production of memory T cells, and additionally induced tumor-specific CTL responses and IFN- $\gamma$ secretion. The development of immunologic memory was demonstrated by challenge of HCC mice with the same tumor cells. In conclusion, the present study investigated the potential anticancer effects of a recombinant adenovirus expressing IL-2. The results of the present study suggested that rAd-IL-2 therapy represents an attractive novel agent for the treatment of HCC, and other cancer types, in future clinical trials.

\section{References}

1. Fung SK and Lok AS: Management of patients with hepatitis B virus-induced cirrhosis. J Hepatol 42 (Suppl): S54-S64, 2005

2. Huang YH, Wu JC, Chen SC, Chen $\mathrm{CH}$, Chiang JH, Huo TI, Lee PC, Chang FY and Lee SD: Survival benefit of transcatheter arterial chemoembolization in patients with hepatocellular carcinoma larger than $10 \mathrm{~cm}$ in diameter. Aliment Pharmacol Ther 23: 129-135, 2006

3. Lubienski A, Bitsch RG, Schemmer P, Grenacher L, Düx M and Kauffmann GW: Long-term results of interventional treatment of large unresectable hepatocellular carcinoma (HCC): Significant survival benefit from combined transcatheter arterial chemoembolization (TACE) and percutaneous ethanol injection (PEI) compared to TACE monotherapy. Rofo 176: 1794-1802, 2004 (In German).

4. Yeh ML, Huang CI, Huang CF, Hsieh MY, Huang JF, Dai CY, Lin ZY, Chen SC, Yu ML and Chuang WL: Neoadjuvant transcatheter arterial chemoembolization does not provide survival benefit compared to curative therapy alone in single hepatocellular carcinoma. Kaohsiung J Med Sci 31: 77-82, 2015. 
5. Marabelle A and Gray J: Tumor-targeted and immune-targeted monoclonal antibodies: Going from passive to active immunotherapy. Pediatr Blood Cancer 62: 1317-1325, 2015.

6. Nishimura Y, Tomita Y, Yuno A, Yoshitake Y and Shinohara M Cancer immunotherapy using novel tumor-associated antigenic peptides identified by genome-wide cDNA microarray analyses. Cancer Sci 106: 505-511, 2015.

7. Hemminki O, Parviainen S, Juhila J, Turkki R, Linder N, Lundin J, Kankainen M, Ristimäki A, Koski A, Liikanen I, et al: Immunological data from cancer patients treated with Ad5/3-E2F- $224-$ GMCSF suggests utility for tumor immunotherapy. Oncotarget 6: 4467-4481, 2015.

8. Smith SG, Koppolu BP, Ravindranathan S, Kurtz SL, Yang L, Katz MD and Zaharoff DA: Intravesical chitosan/interleukin-12 immunotherapy induces tumor-specific systemic immunity against murine bladder cancer. Cancer Immunol Immunother 64 689-696, 2015

9. Bai FL, Tian H, Yu QZ, Renl GP and Li DS: Expressing foreign genes by Newcastle disease virus for cancer therapy. Mol Biol (Mosk) 49: 195-204, 2015 (In Russian).

10. Snyder A, Zamarin D and Wolchok JD: Immunotherapy of Melanoma. Prog Tumor Res 42: 22-29, 2015.

11. Bai FL, Yu YH, Tian H, Ren GP, Wang H, Zhou B, Han XH, Yu QZ and Li DS: Genetically engineered Newcastle disease virus expressing interleukin-2 and TNF-related apoptosis-inducing ligand for cancer therapy. Cancer Biol Ther 15: 1226-1238, 2014

12. Shi L, Zhou Q, Wu J, Ji M, Li G, Jiang J and Wu C: Efficacy of adjuvant immunotherapy with cytokine-induced killer cells in patients with locally advanced gastric cancer. Cancer Immunol Immunother 61: 2251-2259, 2012.

13. Chen Y, Guo ZQ, Shi CM, Zhou ZF, Ye YB and Chen Q: Efficacy of adjuvant chemotherapy combined with immunotherapy with cytokine-induced killer cells for gastric cancer after d2 gastrectomy. Int J Clin Exp Med 8: 7728-7736, 2015.

14. Lippitz BE: Cytokine patterns in patients with cancer: A systematic review. Lancet Oncol 14: e218-e228, 2013.

15. Guan YS, Liu Y, He Q, Li X, Yang L, Hu Y and La Z: p53 gene therapy in combination with transcatheter arterial chemoembolization for HCC: One-year follow-up. World J Gastroenterol 17: 2143-2149, 2011.

16. Graepler F, Verbeek B, Graeter T, Smirnow I, Kong HL, Schuppan D, Bauer M, Vonthein R, Gregor M and Lauer UM: Combined endostatin/sFlt-1 antiangiogenic gene therapy is highly effective in a rat model of HCC. Hepatology 41: 879-886, 2005 .

17. Zou W, Luo C, Zhang Z, Liu J, Gu J, Pei Z, Qian C and Liu X: A novel oncolytic adenovirus targeting to telomerase activity in tumor cells with potent. Oncogene 23: 457-464, 2004.

18. Hernández-Alcoceba R, Sangro B and Prieto J: Gene therapy of liver cancer. Ann Hepatol 6: 5-14, 2007.

19. Yan F, Zheng Y and Huang L: Adenovirus-mediated combined anti-angiogenic and pro-apoptotic gene therapy enhances antitumor efficacy in hepatocellular carcinoma. Oncol Lett 5 : 348-354, 2013

20. Greaves MF and Brown G: Purification of human T and B lymphocytes. J Immunol 112: 420-423, 1974.

21. Zamarin D, Vigil A, Kelly K, García-Sastre A and Fong Y: Genetically engineered Newcastle disease virus for malignant melanoma therapy. Gene Ther 16: 796-804, 2009.

22. Sinkovics JG and Horvath JC: Natural and genetically engineered viral agents for oncolysis and gene therapy of human cancers. Arch Immunol Ther Exp (Warsz) 56 (Suppl 1): 3S-59S, 2008

23. Zhang MM, Yan LN, Li DH, Gou XH, Liu JW, Su Z, Han L and Zhao LY: Inhibition of adenovirus-mediated gene transfer of antisense matrix metalloproteinase-2 on hepatocellular carcinoma growth in vivo. Zhonghua Gan Zang Bing Za Zhi 13: 671-674, 2005 (In Chinese).
24. Ye Z, Wang X, Hao S, Zhong J, Xiang J and Yang J: Oncolytic adenovirus-mediated E1A gene therapy induces tumor-cell apoptosis and reduces tumor angiogenesis leading to inhibition of hepatocellular carcinoma growth in animal model. Cancer Biother Radiopharm 21: 225-234, 2006.

25. Hu HY, Xue XB, Chen K, Wang CJ, Li Y, Zheng JW, Yu Y, Ji WW and $\mathrm{Wu} \mathrm{ZD}$ : Inhibition of hepatocellular carcinoma growth by adenovirus-mediated mda-7 with adriamycin. Zhonghua Gan Zang Bing Za Zhi 16: 461-462, 2008 (In Chinese).

26. He L, Gong HX, Li XP, Wang YD, Li Y, Huang JJ, Xie D, Kung HF and Peng Y: Inhibition of hepatocellular carcinoma growth by adenovirus-mediated expression of human telomerase reverse transcriptase $\mathrm{COOH}-27$ terminal polypeptide in mice. Oncol Lett 6: 748-752, 2013.

27. Thomas AA, Ernstoff MS and Fadul CE: Immunotherapy for the treatment of glioblastoma. Cancer J 18: 59-68, 2012.

28. Larsen CJ: Cellular immunotherapy and glioblastoma: A hopeful treatment?. Bull Cancer 98: 457, 2011 (In French).

29. Varghese S, Rabkin SD, Nielsen GP, MacGarvey U, Liu R and Martuza RL: Systemic therapy of spontaneous prostate cancer in transgenic mice with oncolytic herpes simplex viruses. Cancer Res 67: 9371-9379, 2007.

30. Husain SR, Behari N, Kreitman RJ, Pastan I and Puri RK: Complete regression of established human glioblastoma tumor xenograft by interleukin- 4 toxin therapy. Cancer Res 58 3649-3653, 1998

31. Debinski W, Gibo DM, Obiri NI, Kealiher A and Puri RK: Novel anti-brain tumor cytotoxins specific for cancer cells. Nat Biotechnol 16: 449-453, 1998.

32. Bera TK, Viner J, Brinkmann E and Pastan I: Pharmacokinetics and antitumor activity of a bivalent disulfide-stabilized $\mathrm{FV}_{\mathrm{V}}$ immunotoxin with improved antigen binding to erbB2. Cancer Res 59: 4018-4022, 1999.

33. Ghetie MA, Richardson J, Tucker T, Jones D, Uhr JW and Vitetta ES: Antitumor activity of Fab' and IgG-anti-CD22 immunotoxins in disseminated human B lymphoma grown in mice with severe combined immunodeficiency disease: Effect on tumor cells in extranodal sites. Cancer Res 51: 5876-5880, 1991.

34. Wang X, Bayer ME, Chen X, Fredrickson C, Cornforth AN, Liang G, Cannon J, He J, Fu Q, Liu J, et al: Phase I trial of active specific immunotherapy with autologous dendritic cells pulsed with autologous irradiated tumor stem cells in hepatitis B-positive patients with hepatocellular carcinoma. J Surg Oncol 111: 862-867, 2015

35. Kusnierczyk H, Pajtasz-Piasecka E, Koten JW, Bijleveld C, Krawczyk K and Den Otter W: Further development of local IL-2 therapy of cancer: Multiple versus single IL-2 treatment of transplanted murine colon carcinoma. Cancer Immunol Immunother 53: 445-452, 2004.

36. Pantuck AJ and Belldegrun AS: Phase I clinical trial of interleukin 2 (IL-2) gene therapy for prostate cancer. Curr Urol Rep 2: 33, 2001.

37. Baek S, Kim YM, Kim SB, Kim CS, Kwon SW, Kim Y, Kim H and Lee H: Therapeutic DC vaccination with IL-2 as a consolidation therapy for ovarian cancer patients: A phase I/II trial. Cell Mol Immunol 12: 87-95, 2015.

38. Tan Y, Xu M, Wang W, Zhang F, Li D, Xu X, Gu J and Hoffman RM: IL-2 gene therapy of advanced lung cancer patients. Anticancer Res 16: 1993-1998, 1996.

39. Bai F, Niu Z, Tian H, Li S, Lv Z, Zhang T, Ren G and Li D: Genetically engineered Newcastle disease virus expressing interleukin 2 is a potential drug candidate for cancer immunotherapy. Immunol Lett 159: 36-46, 2014. 